\title{
Novel micromachined silicon acoustic delay line systems for real-time photoacoustic tomography applications
}

C.-C. Chang, Y. Cho, L. V. Wang, J. Zou

C.-C. Chang, Y. Cho, L. V. Wang, J. Zou, "Novel micromachined silicon acoustic delay line systems for real-time photoacoustic tomography applications," Proc. SPIE 8581, Photons Plus Ultrasound: Imaging and Sensing 2013, 85813B (4 March 2013); doi: 10.1117/12.2004573

SPIE. Event: SPIE BiOS, 2013, San Francisco, California, United States 


\title{
Novel micromachined silicon acoustic delay line systems for real-time photoacoustic tomography applications
}

\author{
C.-C. Chang ${ }^{* a}$, Y. Cho a , L. V. Wang ${ }^{b}$, J. Zou ${ }^{\mathrm{a}}$ \\ ${ }^{\mathrm{a}}$ Dept. of Electrical and Computer Engineering, Texas A\&M University, 3128 TAMU, College

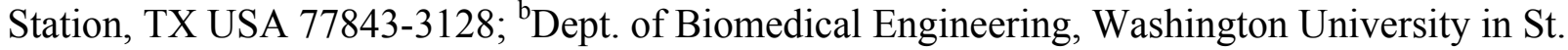 \\ Louis, 1 Brookings Dr., St. Louis, MO USA 63130
}

\begin{abstract}
In current photoacoustic tomography (PAT) systems, ultrasound transducer arrays and multi-channel data acquisition (DAQ) electronics are used to receive the PA signals. To achieve real-time PA imaging, massive 1D or even 2D transducer arrays and large number of DAQ channels are necessary. As a result, the ultrasound receiver becomes very complex, bulky and also costly. In this paper, we report the development of novel micromachined silicon acoustic delay line systems, which are expected to provide a new approach to address the above issue. First, fundamental building block structures of the acoustic delay line systems were designed and fabricated. Their acoustic properties were characterized with ultrasound and photoacoustic measurements. Second, two different acoustic delay line systems (parallel and serial) were designed and fabricated using advanced micromachining processes to ensure compact size, high accuracy, and good repeatability. The transmission of multiple acoustic signals in the acoustic delay line systems were studied with ultrasound experiment. Experimental results show that the silicon acoustic delay line systems can guide multiple channels of acoustic signals with low loss and distortion. With the addition of a set of suitable time delays, the timedelay acoustic signals arrived at a single-element transducer at different times and were unambiguously received and processed by the following DAQ electronics. Therefore, the micromachined silicon acoustic delay line systems could be used to combine multiple signal channels into a single one (without the involvement of electronic multiplexing), thereby reducing the complexity and cost of the ultrasound receiver for real-time PAT application.
\end{abstract}

Keywords: Photoacoustic tomography, ultrasound, delay line, micromachining.

\section{INTRODUCTION}

In photoacoustic tomography, ultrasound transducer arrays have been used to receive photoacoustic signals from the source point $(\mathrm{s})^{1}$. Each element of the transducer array has a corresponding data acquisition electronic circuit to process the signals (figure 1). To achieve fast speed, high resolution, and real-time photoacoustic imaging, it is inevitable to use massive high-frequency transducer arrays with complex DAQ electronics which function simultaneously ${ }^{2}$. As a result, the entire system could become complex, bulky, and costly.

To address this issue, a novel photoacoustic receiving system design utilizing delay lines to control the time of flight of the signals was developed and demonstrated ${ }^{3}$. Unlike conventional transducer array using multiple transducer elements, the delay lines replace the transducer elements and connect with a single receiving transducer (figure 2). Each delay line receives the photoacoustic signals and introduces proper time delay for them to reach the single transducer. Therefore, signals from multiple channels processed by multiple DAQ electronics at the same time are now converted to a series of signals that can be processed serially in single channel DAQ electronics. This new approach could provide a more economical approach for constructing photoacoustic receiving systems.

Delay lines made by different materials such as liquid mercury ${ }^{4}$, quartz $\operatorname{rods}^{5}$, metal wires ${ }^{6}$, and optical fiber ${ }^{7}$ were used in many different applications ${ }^{8}$. The acoustic wave propagation in various geometries of the delay line was also investigated ${ }^{9,10}$. In this paper, we propose a new micromachined silicon delay line system. The system has an extremely low acoustic loss from the single-crystalline silicon and is compatible to the micromachining process with high precision. We first investigate acoustic propagation in delay lines with different geometries. Based on the result, two silicon delay line systems, parallel and serial, were designed and fabricated to demonstrate the controlled time delays in multiple channels of acoustic signals with a single element transducer.

Photons Plus Ultrasound: Imaging and Sensing 2013, edited by Alexander A. Oraevsky, Lihong V. Wang, Proc. of SPIE Vol. 8581, 85813B - (C) 2013 SPIE · CCC code: 1605-7422/13/\$18 - doi: 10.1117/12.2004573 

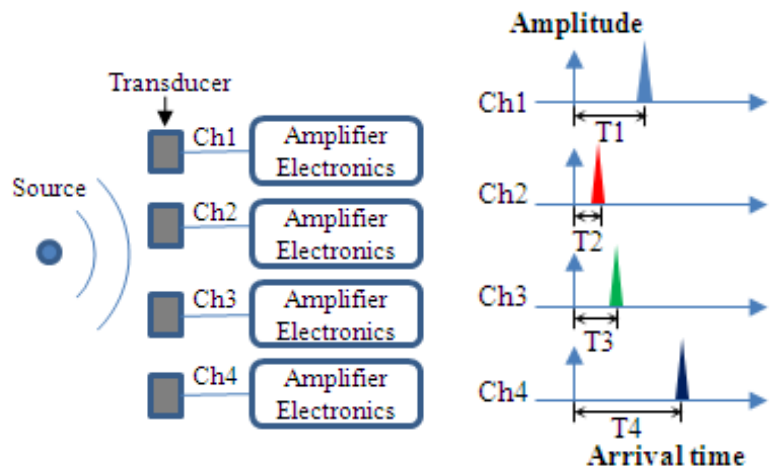

Figure 1. Ultrasound transducer array. Left: Array setup; Right: Signals received by transducer. The arrival time is the travel time of the signal in the medium.
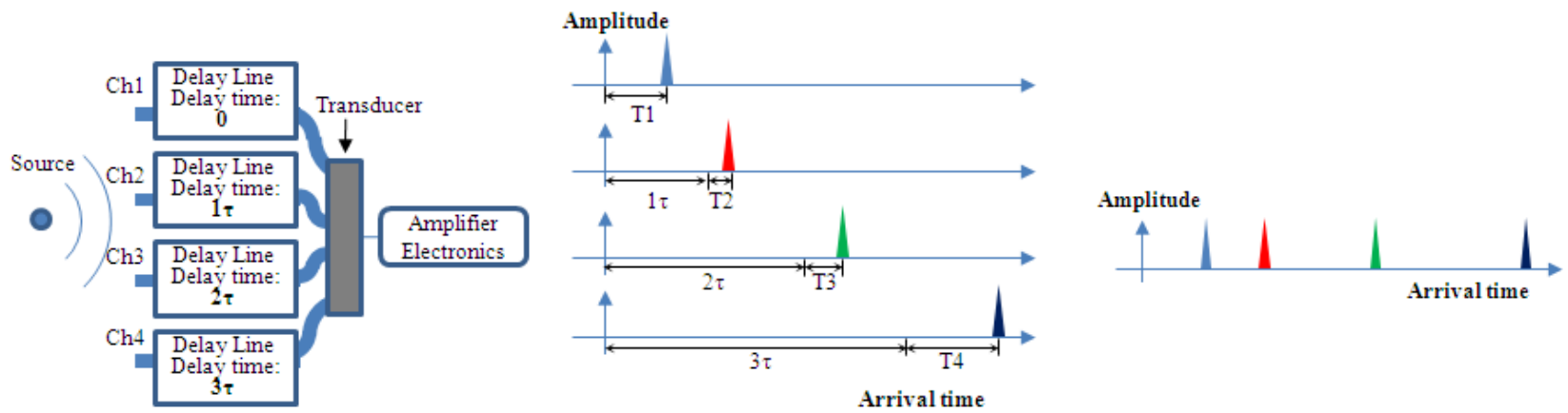

Figure 2. Delay line system. Top left: Delay lines with single transducer; Top right: Time-delayed ultrasound signals; Bottom: Signals received by the single transducer.

\section{DESI* N AND FABRICATION}

Silicon is desirable for the following reasons. First, the acoustic attenuation of silicon in the MHz range is extremely low $\left(\sim 10^{-4} \mathrm{~dB} / \mathrm{mm} \cdot \mathrm{MHz}^{2} @ 10 \mathrm{MHz}\right)^{11}$. Low attenuation implies a high transmission efficiency of the delay line. The dispersion of the acoustic wave can be controlled by changing the dimensions of the delay line ${ }^{12}$. Second, silicon is a strong material and has high thermal stability. Last, microfabrication and micromachining technologies for silicon have been well-developed. Different silicon delay line designs can be fabricated precisely at the same time with minimal assembly.

The dispersion of the acoustic wave is affected by the dimension of the delay line. To minimize the dispersion effect, the acoustic wave should be operated in the lowest longitudinal mode. For a rectangular-shaped delay line, this can be achieved by setting the delay line dimension to satisfy $\left(d f / V_{0}\right)<<1$, where $d$ is the width or thickness of the delay line (whichever is smaller), $f$ is the frequency of the signal, and $V_{0}$ is the acoustic velocity of the delay line material ${ }^{12}$ (for silicon, $8430 \mathrm{~m} / \mathrm{s}^{13}$ ). In this paper, all the delay lines were designed to have $500 \mu \mathrm{m}$ in width and $250 \mu \mathrm{m}$ in thickness to transmit $2.25 \mathrm{MHz}$ acoustic signals under single mode.

In the delay line system, each channel has a specific physical length, which determines the delay time of the signal it carries. Based on that, the silicon delay line can have two different layouts, parallel and serial (figure 3). In the parallel layout, each channel functions independently and has a specific length and time delay. All channels connect to the same transducer at the distance ends. Since each delay line has a different length, the acoustic signals will arrive at the receiving transducer at different time. Due to the high acoustic velocity of silicon, it is inevitable to use long silicon delay line for sufficient time delay. This can be achieved by using multiple different radii U-turn structures winding together in a compact space. In the serial layout, all channels merge into the main channel with Y-shape junctions. The length difference for each channel is determined by the merging location. The signal travelling length inside the main channel controls the delay time. 


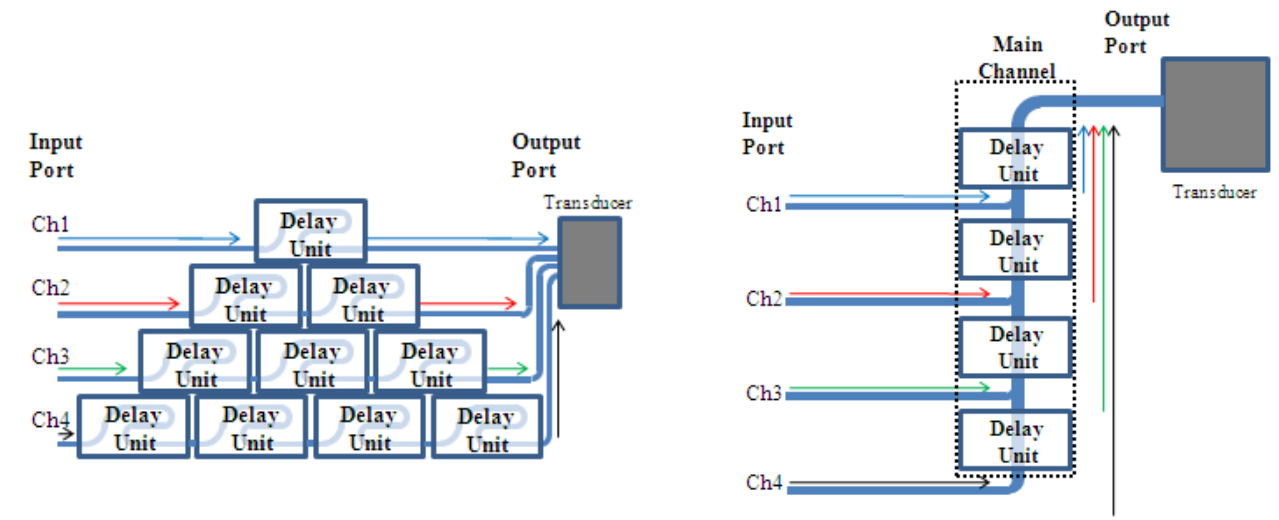

Figure 3. Delay line layouts. Left: Schematic of parallel delay lines; Right: Schematic of serial delay lines.

The delay line structures were fabricated using 4-inch single crystalline silicon wafer with a thickness of $250 \mathrm{um}$. The wafer was coated with aluminum. The aluminum layer was patterned by photolithography and used as a protective mask for the later silicon etching. A cryogenic deep reaction process ${ }^{14}$ was used to etch out the silicon delay line structures. After etching, the delay line structures were carefully released from the wafer holder.

\section{EXPERIMENTAL METHOD}

Figure 4 shows the characterization setup. The silicon samples are held on the acrylic holding structures, together with a $2.25 \mathrm{MHz}$ longitudinal wave transducer attached on one end of the silicon samples. The ends of the silicon samples are well polished and mineral oil is applied to enhance the coupling efficiency between the silicon sample and the transducer. The whole assembly is fixed on a three axis translation stage. For acoustic signal excitation, photoacoustic effect ${ }^{15}$ is used to generate acoustic wave directly inside the silicon samples. The laser used is an attenuated $1064 \mathrm{~nm}$ wavelength Nd:YAG laser. The laser has a $10 \mathrm{~Hz}$ repetition rate for single pulse excitation and 10 nanosecond pulse width. Silicon absorbs the light energy and creates a pressure wave originated from the excitation point. The pressure wave travels along the silicon delay line and is detected by the transducer. The signal is further amplified by the pulser-receiver unit and displayed on the oscilloscope. The result is averaged from multiple data points for each measurement.

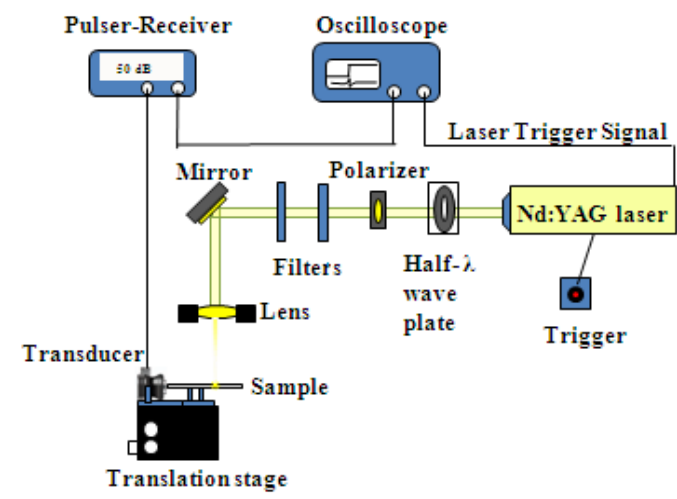

Figure 4. Schematic of photoacoustic excitation setup. The laser is attenuated and controlled by the trigger.

\section{RESULT}

\subsection{Acoustic properties: attenuation and velocity}

The acoustic attenuation and velocity were measured using a straight silicon delay line with a length of $7 \mathrm{~cm}$. A focused laser light was illuminated at a particular location on the silicon delay line (figure 5). Pressure wave was generated from the photoacoustic effect and traveled toward the receiving transducer. Wave travelling away from the transducer was reflected back on the other end and reached the same transducer. The laser excitation spots have a small distance away from both ends of the delay line to avoid the reflected signals to overlap with the original one. In the experiment, the 
excitation points were located on eight different spots with approximately $5 \mathrm{~mm}$ interval along the silicon delay line. From the ratio of travelling length and signal arrival time, the acoustic velocity of a straight silicon line is calculated to be $\sim 8454 \mathrm{~m} / \mathrm{s}$. From the relation between receiving signal amplitudes and travelling length, the attenuation is almost negligible for 2.25 MHZ signal (figure 6).

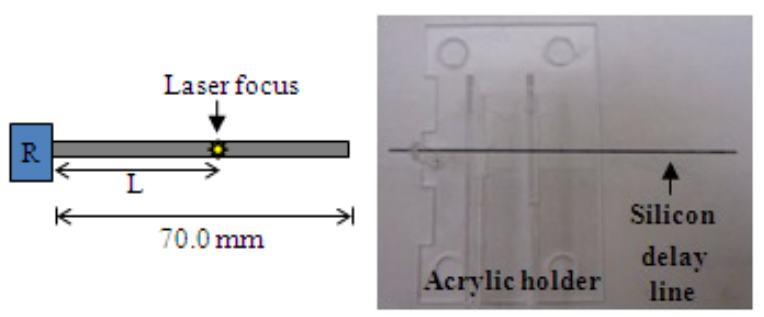

Figure 5. Silicon delay line setup for acoustic velocity and attenuation measurement. Left: Schematic of the straight delay line and measurement setup. L: Travel length of the acoustic signal. R: Receiving transducer; Right: Fabricated straight silicon delay line placed on an acrylic holder.
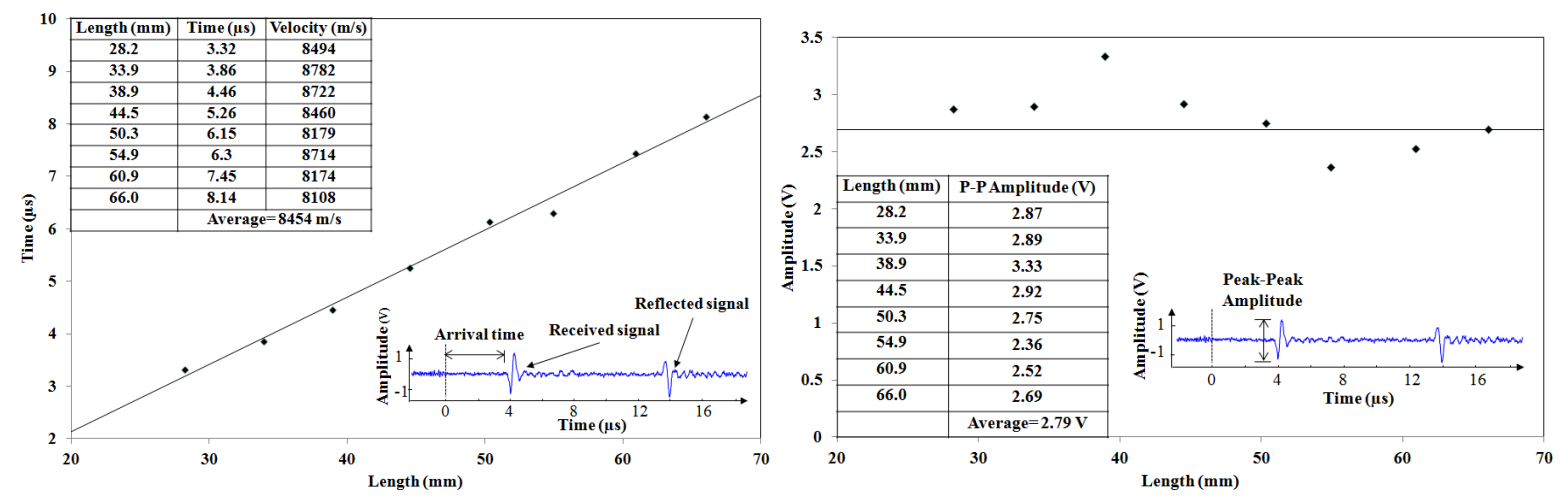

Figure 6. Left: Plot of the arrival time versus traveling distance for determining the acoustic velocity; Right: Plot of the signal peak-peak amplitude versus traveling length to determine the acoustic attenuation. Inset: Representative signal shape from L: $28.2 \mathrm{~mm}$.

\subsection{Propagation in U-turn bending structures with different radii}

To investigate the acoustic wave transmission in U-turn structures, six bending silicon delay lines with different radii of curvature were used in the measurement. The bending silicon delays are shown in the figure 7, with two straight portion connected by a $180^{\circ}$ U-turn bend. The laser excitation spots were located at the junction between the straight part and the bending part. At point A, the signal only travels in the straight part. In point B, the signal travels an additional bending part. By comparing signals generated from both junctions, the signal difference due to bending can be determined. From the experimental results, the signal amplitude ratio between point B and point A decreases as the radius decreases (figure 8). This is due to the mode conversion and reflection loss of the acoustic wave.
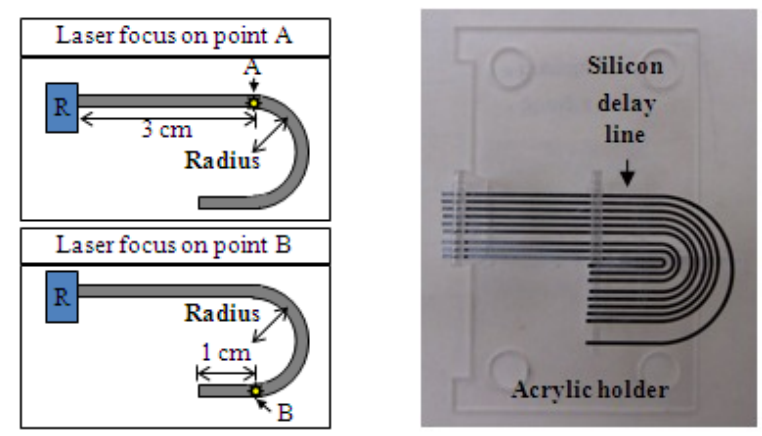

Figure 7. U-turn structure measurement. Left: Schematic of the U-turn structure and measurement setup. A: Without bending. B: With bending; Right: Fabricated U-turn delay line structures. 


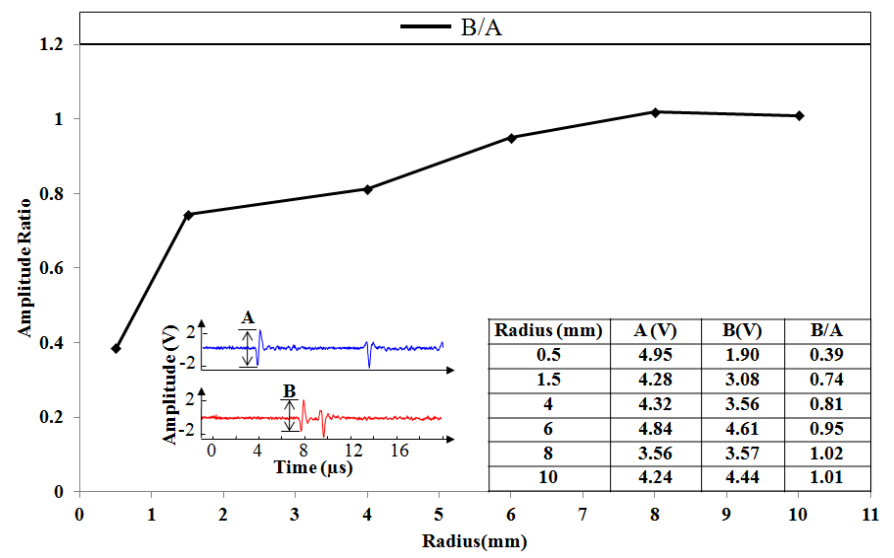

Figure 8. Plot of the signal peak-peak amplitude ratio at point B and point A versus bending radius. Inset: Representative signal shape from $6 \mathrm{~mm}$ radius structure.

\subsection{Propagation in Y-junction structures with different merging angles}

Six Y-junction structures were used to investigate the cross angle effect on signal. The angles ranged from 5 degrees to 30 degrees with a 5-degree increment. Each structure has a 4-cm straight silicon line merged with a 3-cm-long straight silicon line located at $1 \mathrm{~cm}$ away from the transducer. The laser excitation spots were located on the two branches and the intersection point (figure 9). Signals from the two branches were affected by the junction, while signal generated from the junction point was not. By comparing the signal amplitude from these three points, the insertion loss due to the junction can be determined. From the experimental results, the junction angle difference from 5 to 30 degrees doesn't have a significant effect on the insertion loss. The loss is due to the wave leakage, and the average loss in the experiment is around $20 \%$ (figure 10 ).
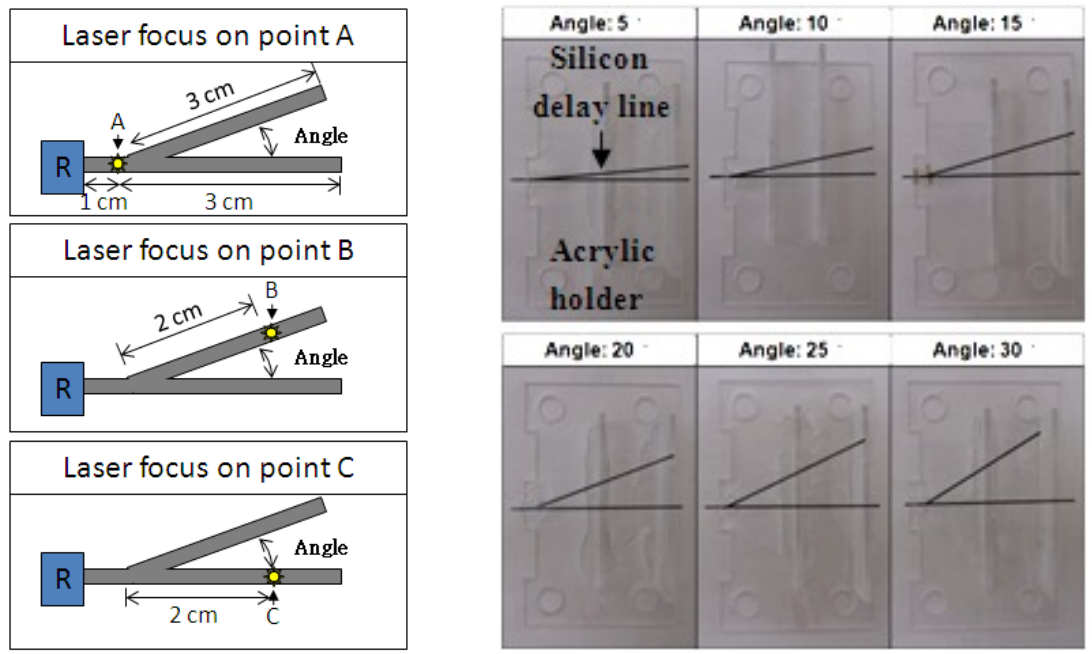

Figure 9. Y-junction measurement. Left: Schematic of the Y-junction structure and measurement setup. A: Without passing junction. B: Passing junction with angle through branch. C: Passing junction through straight line; Right: Fabricated YJunction delay line structures 


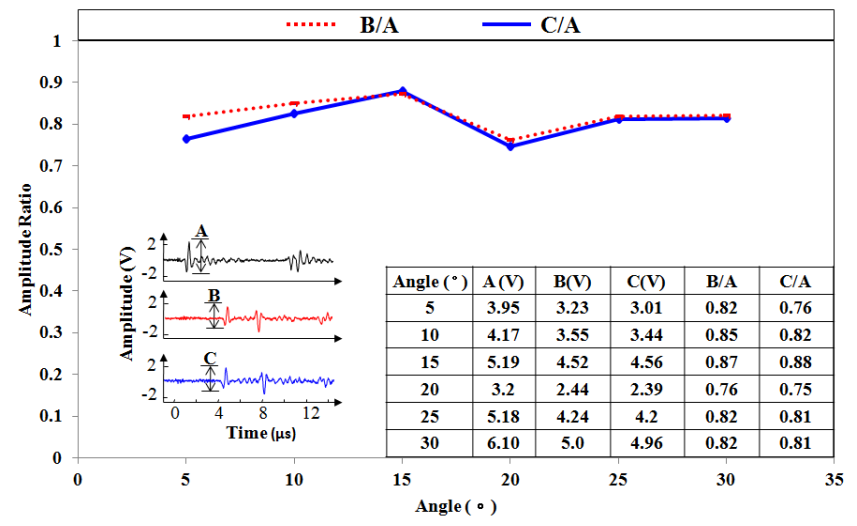

Figure 10. Plot of the signal peak-peak amplitude ratio versus different merging angles. Inset: Representative signal shape from junction with 15 degrees.

\section{SERIAL AND PARALLEL DELAY LINE}

Based on the experimental results of the fundamental structures of the delay line, a 4-channle serial delay lines and a 4channel parallel delay lines (figure 11) were designed, fabricated and tested with a two-port through transmission setup (figure 12). The delay lines were placed on the acrylic holder. The input and output ports were polished and in contact with the transmitting and receiving transducers respectively. A pulser-receiver unit was used to drive the $2.25 \mathrm{MHz}$ transmitting transducer and amplify the received signal. Signals from each channel traveled different length and arrived at the output transducer at different time points. The serial delay line is made of straight lines connected with a $4 \mathrm{~mm}$ radius U-turn structures, with 30-degree Y-junctions for channel merging. It has four inputs and one output. The parallel delay line is made of multiple spiral structures, which have minimum radius of $2.5 \mathrm{~mm}$. It has four inputs and four outputs.
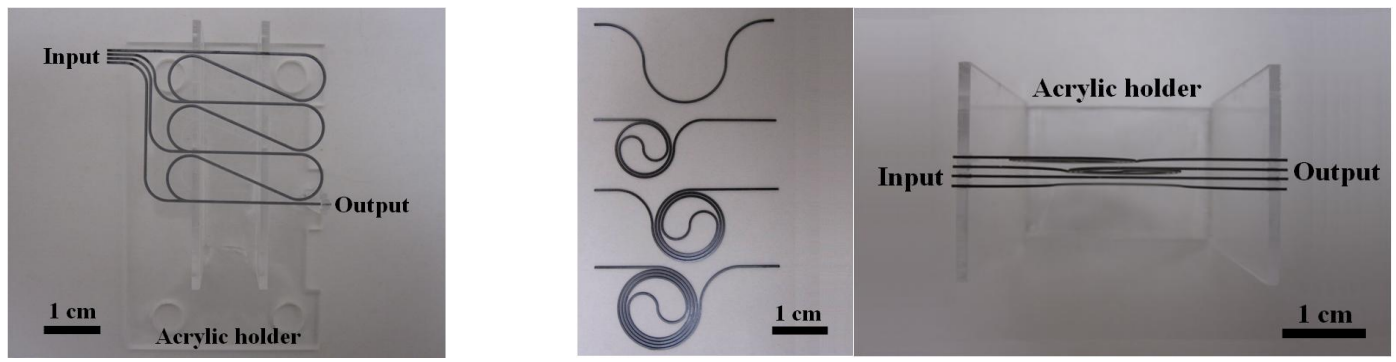

Figure 11. Fabricated 4-channel delay line systems. Left: Serial delay line. Right: Parallel delay line assembled on acrylic holder.

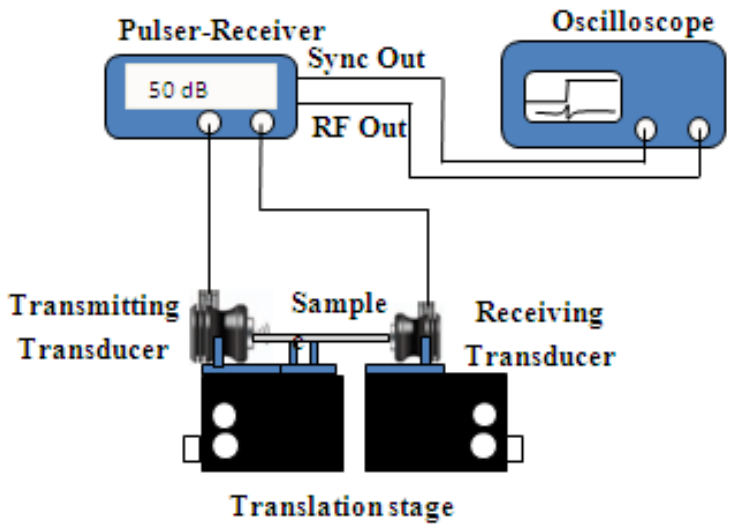

Figure 12. Two-port through transmission setup. 
The received signals from the serial and parallel delay line are shown in figure 13. The signals remain clear and distinguishable. In both layouts, a $7 \mu$ s delay interval were successfully achieved for all four channels. The signal amplitude decreases from channel 1 to channel 4. The decrease in amplitude is from the transmission and insertion loss of the U-turn and Y-junction structures. Since longer channel has more U-turns and Y-junctions, the total insertion loss is higher and the signal is smaller.
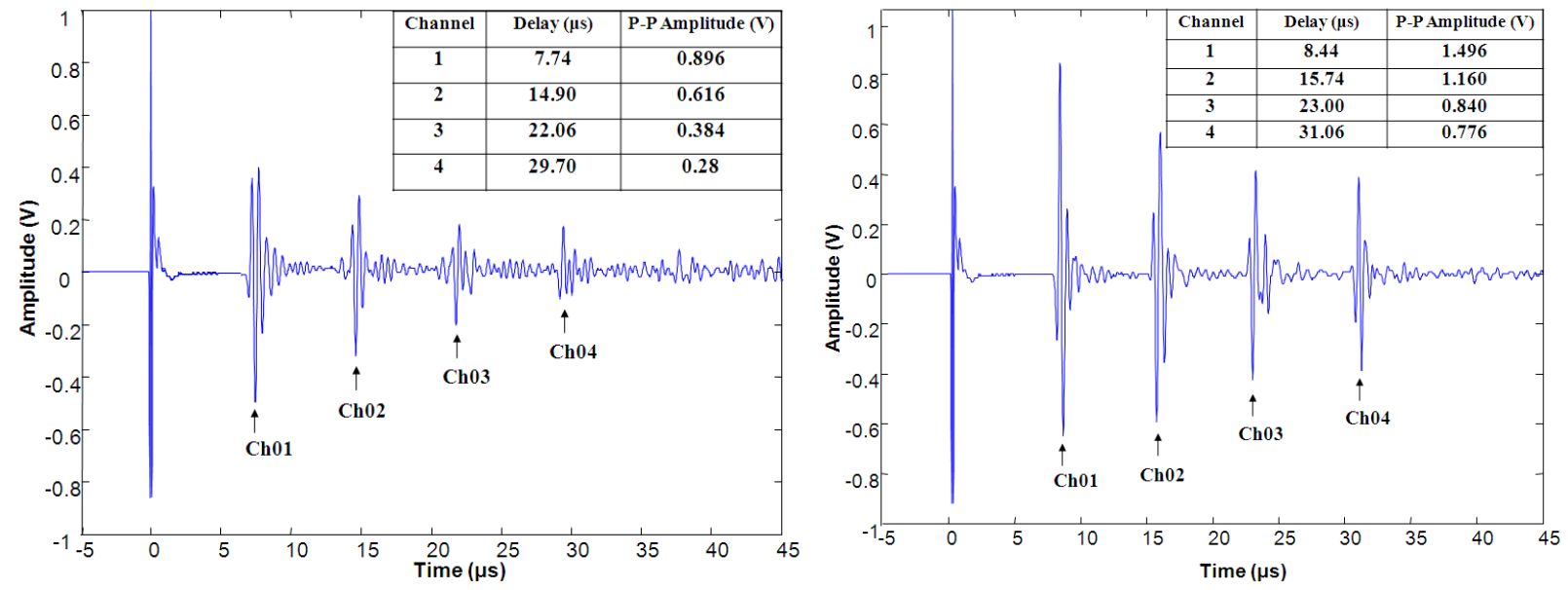

Figure 13. Signal from the 4-channel delay lines. Left: From the serial delay lines; Right: From the parallel delay lines.

\section{CONCLUSION}

In this work, we have designed, fabricated, and tested a novel micromachined silicon-based acoustic delay line system. The acoustic properties of silicon delay line structures have been characterized and the acoustic wave propagation in Uturn and Y-junction structures has been experimentally tested. Two delay line layouts, parallel and serial, have been realized and are able to achieve different time delays. Multiple channels of parallel signals therefore can be converted into single channel of serial time-delayed signals. By carefully design and construct the delay lines, acoustic signals can be distinctive with minimum attenuation and distortion. As a result, we believe the micromachined silicon delay lines can be used to reduce the complexity and cost of the ultrasound receiving system in photoacoustic tomography.

\section{REFERENCES}

[1] Wang, L. V., "Tutorial on photoacoustic microscopy and computer tomography," IEEE J. Sel. Top. Quant. Electron. 14(1), 171-179 (2008).

[2] Gamelin. J., Maurudis, A., Aguirre, A., Huang, F., Guo, P. Y., Wang, L. V. and Zhu, Q., "A real-time photoacoustic tomography system for small animals," Opt. Express 17(13), 10489-10498 (2009).

[3] Yapici, M. K., Kim, C., Chang, C.-C., Jeon, M., Guo, Z., Cai, X., Zou, J. and Wang, L. V., "Parallel acoustic delay lines for photoacoustic tomography," J. Biomed. Opt. 17(11), 116019 (2012).

[4] Auerbach, I. L., Echert, J. P., Shaw, R. F. and Sheppard, C. B., "Mercury delay line memory using a pulse rate of several megacycles," Pro. Inst. Radio Eng. 37(8), 855-861 (1949).

[5] Arenberg, D. L., "Ultrasonic solid delay lines," J. Acoust. Soc. Am. 20(1), 1-26 (1948).

[6] Scarrott, G. G. and Naylor, R., "Wire-type acoustic delay lines for digital storage," Proc. of the IEE - Part B: Radio and Electron Eng. 103(3), 497-508 (1956).

[7] Boyd, G. D., Coldren, L. A. and Thurston, R. N., "Acoustic clad fiber delay-lines," IEEE Trans. Son. Ultrason. 24(4), 24-252 (1977).

[8] Palfreeman, J. S., "Acoustic delay lines - a survey of types and uses," Ultrasonics 3(1), 1-8 (1965).

[9] Meitzler, A. H., "Ultrasonic delay lines for digital data storage," IRE Trans. Ultrason. Eng. 9(2), 30-37 (1961).

[10] Gibson, R. W., "Solid ultrasonic delay lines," Ultrasonics 3(2), 49-61 (1965).

[11] Auld, B. A., [Acoustic fields and waves in solids], Charles Krieger, Florida, USA (1991). 
[12] Meeker, T. R., "Dispersive ultrasonic delay lines using the first longitudinal mode in a strip," IRE Trans. Ultrason. Eng. 7(2), 53-58 (1960).

[13]David, J. and Cheeke, N., [Fundamentals and applications of ultrasonic waves], CRC Press, Montreal, Canada (2002).

[14]De Boer, M. J., Gardeniers, J. G. E., Jansen, H. V., Smulders, E., Gilde, M. J., Roelofs, G., Sasserath, J. N. and Elwenspoek, M., "Guidelines for etching silicon MEMS structures using fluorine high-density plasmas at cryogenic temperatures," J. Microelectromech. Syst. 11(4), 385-401 (2002).

[15]Rosencwaig, A. and Gersho, A., "Theory of the photoacoustic effect with solids," J. Appl. Phys. 47(1), 64-69 (1976). 